\title{
Effective accuracy of stereeelectroencephalography: robotic 3D versus Talairach orthogonal approaches
}

\author{
*Pierre Bourdillon, MD,1-4 Claude-Edouard Châtillon, MD, PhD, ${ }^{5,6}$ Alexis Moles, MD,1 \\ Sylvain Rheims, MD, PhD, ${ }^{2,7,8}$ Hélène Catenoix, MD, ${ }^{7}$ Alexandra Montavont, MD, ${ }^{7}$ \\ Karine Ostrowsky-Coste, MD, ${ }^{7}$ Sebastien Boulogne, MD, ${ }^{2,7}$ Jean Isnard, MD, PhD, ${ }^{7}$ and \\ Marc Guénot, MD, PhD $1,2,9$
}

\begin{abstract}
1'Department of Neurosurgery, Neurology \& Neurosurgery Hospital Pierre Wertheimer, Hospices Civils de Lyon, Lyon, France; ${ }^{2}$ Faculty of Medicine Claude Bernard, University of Lyon, Lyon, France; ${ }^{3}$ Faculty of Science \& Engineering, Sorbonne University, Paris, France; ${ }^{3 B}$ rain and Spine Institute, INSERM U1127, CNRS 7225, Paris, France; ${ }^{5}$ Department of Surgery, Service of Neurosurgery, Centre Hospitalier Affilié Universitaire Régional, Trois-Rivières Hospital, Trois-Rivières, Quebec, Canada; ${ }^{6}$ Faculty of Medicine, Division of Neurosurgery, Université de Montréal, Montreal, Quebec, Canada; ${ }^{7}$ Department of Functional Neurology and Epileptology, Neurology \& Neurosurgery Hospital Pierre Wertheimer, Hospices Civils de Lyon, Lyon, France; ${ }^{8}$ TIGER, Neuroscience Research Center of Lyon, INSERM U1028, CNRS 5292, Université de Lyon, Lyon, France; and ${ }^{9}$ NEUROPAIN Team, Lyon Neuroscience Research Center, INSERM U1028, CNRS 5292, Université de Lyon, Lyon, France
\end{abstract}

\begin{abstract}
OBJECTIVE Stereoelectroencephalography (SEEG) was first developed in the 1950s by Jean Talairach using 2D angiography and a frame-based, orthogonal approach through a metallic grid. Since then, various other frame-based and frameless techniques have been described. In this study the authors sought to compare the traditional orthogonal Talairach 2D angiographic approach with a frame-based 3D robotic procedure that included 3D angiographic interoperative imaging guidance. MRI was used for both procedures during surgery, but MRI preplanning was done only in the robotic 3D technique.
\end{abstract}

METHODS All study patients suffered from drug-resistant focal epilepsy and were treated at the same center by the same neurosurgical team. Fifty patients who underwent the 3D robotic procedure were compared to the same number of historical controls who had previously been successfully treated with the Talairach orthogonal procedure. The effectiveness and absolute accuracy, as well as safety, of the two procedures were compared. Moreover, in the 3D robotic group, the reliability of the preoperative MRI to avoid vascular structures was evaluated by studying the rate of trajectory modification following the coregistration of the intraoperative 3D angiographic data onto the preoperative MRI-based trajectory plans.

RESULTS Effective accuracy (96.5\% vs $13.7 \%$ ) and absolute accuracy $(1.15 \mathrm{~mm}$ vs $4.00 \mathrm{~mm})$ were significantly higher in the 3D robotic group than in the Talairach orthogonal group. Both procedures showed excellent safety results (no major complications). The rate of electrode modification after 3D angiography was $43.8 \%$, and it was highest for frontal and insular locations.

CONCLUSIONS The frame-based, 3D angiographic, robotic procedure described here provided better accuracy for SEEG implantations than the traditional Talairach approach. This study also highlights the potential safety advantage of trajectory planning using intraoperative frame-based 3D angiography over preoperative MRI alone.

https://thejns.org/doi/abs/10.3171/2018.7.JNS181164

KEYWORDS SEEG; stereotaxy; angiography; drug-resistant epilepsy; safety; accuracy; intracranial recordings

$\mathrm{S}$ INCE the early days of the use of stereotaxy in human medicine, $, 1,2,15$ many scientists have developed specific applications of this procedure in their particular field of interest. ${ }^{19,26}$ Among them, Jean Talairach started working on stereotaxy during the 1950 s, as a logical con- sequence of his studies on brain networks. He had already proved to be passionate about mapping, both extraprofessionally - he had drawn, with René Suttel, a cartography of the Paris underground that was used by the French Resistance during World War $\mathrm{II}^{6}$ - and professionally-he had

ABBREVIATIONS AP = anteroposterior; EEG = electroencephalography; MEG = magnetoencephalography; SEEG = stereoelectroencephalography. SUBMITTED April 26, 2018. ACCEPTED July 16, 2018.

INCLUDE WHEN CITING Published online December 7, 2018; DOI: 10.3171/2018.7.JNS181164.

${ }^{*}$ P.B. and C.E.C. contributed equally to this work. 
developed a strong interest in the mapping of brain networks through his primary interest in psychiatry. ${ }^{3}$ Therefore, when Talairach got involved in epilepsy research, he imagined a stereotactic methodology allowing prolonged intracranial electroencephalography (EEG) for recording epileptic networks, namely stereoelectroencephalography (SEEG). He developed this procedure with Gabor Szikla, and the use of angiography to maximize vascular safety by preventing damage to blood vessels was an obsession. ${ }^{27}$ Indeed, after 300 procedures, their methodology appeared to be one of the safest existing techniques for stereotactic invasive surgery, with a reported major complication rate of only $0.3 \% .^{22}$

Recently, 21st-century technical advances have led to major modifications in SEEG. The development of robotics led to increased possibilities in stereotactic surgery, and indications have expanded from purely diagnostic to therapeutic uses, due to the current ability to use the electrodes to create thermolesions in pathological epileptic networks. ${ }^{4,5,12,17}$ These techniques are now widely used, and some studies have reported benefits such as the ability to reach targets obliquely, free of the orthogonal constraints; digitalization of the stereoscopy; and multimodal unification of the stereotactic space, along with increased safety with the application of these advances in SEEG. ${ }^{9-11,23,25}$ Nevertheless, the robotic 3D approach has never been compared, in terms of accuracy, to the Talairach orthogonal procedure. The aim of our study was to provide this direct comparison.

\section{Methods Patients}

All patients who had been successfully treated with an SEEG procedure performed after 2012 were eligible. All of the study patients suffered from drug-resistant focal epilepsy and underwent phase I presurgical evaluations, which consisted of long-term scalp video-EEG monitoring, neuropsychological testing, high-resolution 3-T MRI, magnetic source imaging of interictal paroxysmal activity using magnetoencephalography (MEG), and metabolic imaging (18-fluorodeoxyglucose [ ${ }^{18}$ FDG] PET scan). Some patients also underwent ictal SPECT.

The study participants were divided into 2 groups. The first group included all patients who underwent SEEG after 2015 and therefore benefited from the robotic 3D approach. The second group included the same number of patients, but these patients had benefited from a Talairach orthogonal procedure, performed after 2012 and before 2015 (Fig. 1). All of the procedures in both groups were performed in a single center (Department of Neurosurgery, Neurology \& Neurosurgery Hospital Pierre Wertheimer, Hospices Civils de Lyon, France) by one neurosurgical team (M.G. and P.B.).

Each patient or his/her legal guardian(s) gave informed consent to implantations of the electrodes. This study received the approval of the local ethics committee.

\section{Talairach Orthogonal Approach}

In the Talairach orthogonal group, patients underwent procedures for which the methodology was similar, in principle, to that originally described by Jean Talairach and Gabor Szikla. ${ }^{16,18,27}$ The desirable locations of the targets were identified and discussed with the epileptologists in a multidisciplinary setting, and a schematic map of ideal targets and trajectories, based on 2D sagittal MR images, was produced. In this approach, the preplanning information was not sufficient to calculate stereotactic coordinates, whereas in the robotic 3D approach preplanning was performed in a stereotactic volume with usable spatial coordinates.

On the day of surgery, with the patient under general anesthesia, the patient's head was secured into a Talairach frame and standard anteroposterior (AP) and lateral radiographs were taken to visualize the positioning of the Talairach metallic grids (Fig. 2). A selective Seldinger common carotid artery catheterization was then performed. AP and lateral images were obtained for both arterial and venous phases. In cases of bilateral implantation, this procedure was repeated for the contralateral side.

A manual coregistration was then performed between the patient's sagittal MR images and the 2D angiographic images using shared anatomical landmarks (e.g., by aligning the splenium and genu of the corpus callosum on MRI to the pericallosal arteries and Galen's venous system on angiography). This coregistration was linear and based on rotation, translation, and enlargement factor modifications. Avascular trajectories were selected on the frame-based angiography images in order to always maintain vascular safety, even in the event of deficient coregistration.

The scalp and calvaria were drilled through the Talairach metallic grid holes corresponding to the selected trajectories, and the dura was opened by monopolar coagulation. The screw-in anchors (MRI-compatible titanium; Microdeep, Dixi Medical) were screwed in place and their positions were checked radiographically. Electrode length was determined on AP radiographs, based on the distance between the screw-in anchor and the midline. Electrodes (Microdeep) were then inserted after a first path had been performed with a rigid smooth-tipped stylus. Positioning and depth of the electrodes were checked one last time with an AP radiograph. Postoperative MRI was performed within 24 hours following the surgery.

\section{Robotic 3D Approach}

A few days before surgery, after multidisciplinary discussion of the relevant target locations, presurgical planning of each electrode trajectory, taking into account the vascular anatomy explored by T1 gadolinium-enhanced MRI scans, was performed on Neuroinspire software version 5.0.721.1 (Renishaw PLC).

Then, on the day of surgery, with the patient under general anesthesia, a Leksell frame (Leksell Stereotactic System, Elekta) was placed on the patient's head and a selective Seldinger common carotid artery catheterization was performed. A 3D arterial and venous angiography was then obtained by means of an O-arm II (Medtronic) imaging device, using previously published parameters (Fig. 3). ${ }^{10}$

The 3D angiography was then coregistered with the presurgical planning MR images using a rigid body registration with 3 rotations and 3 translational degrees of 


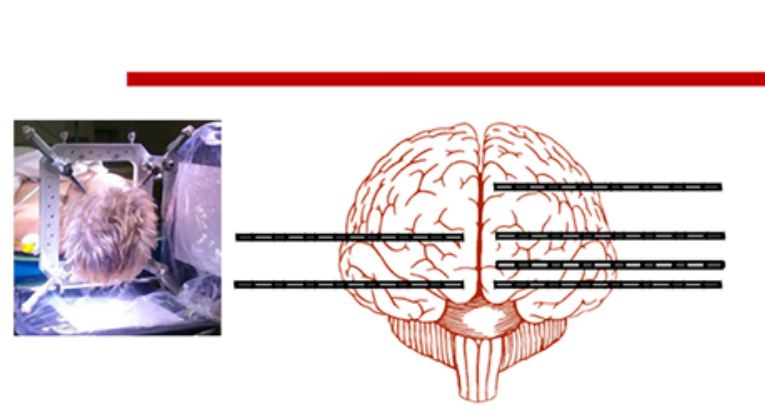

\section{5}
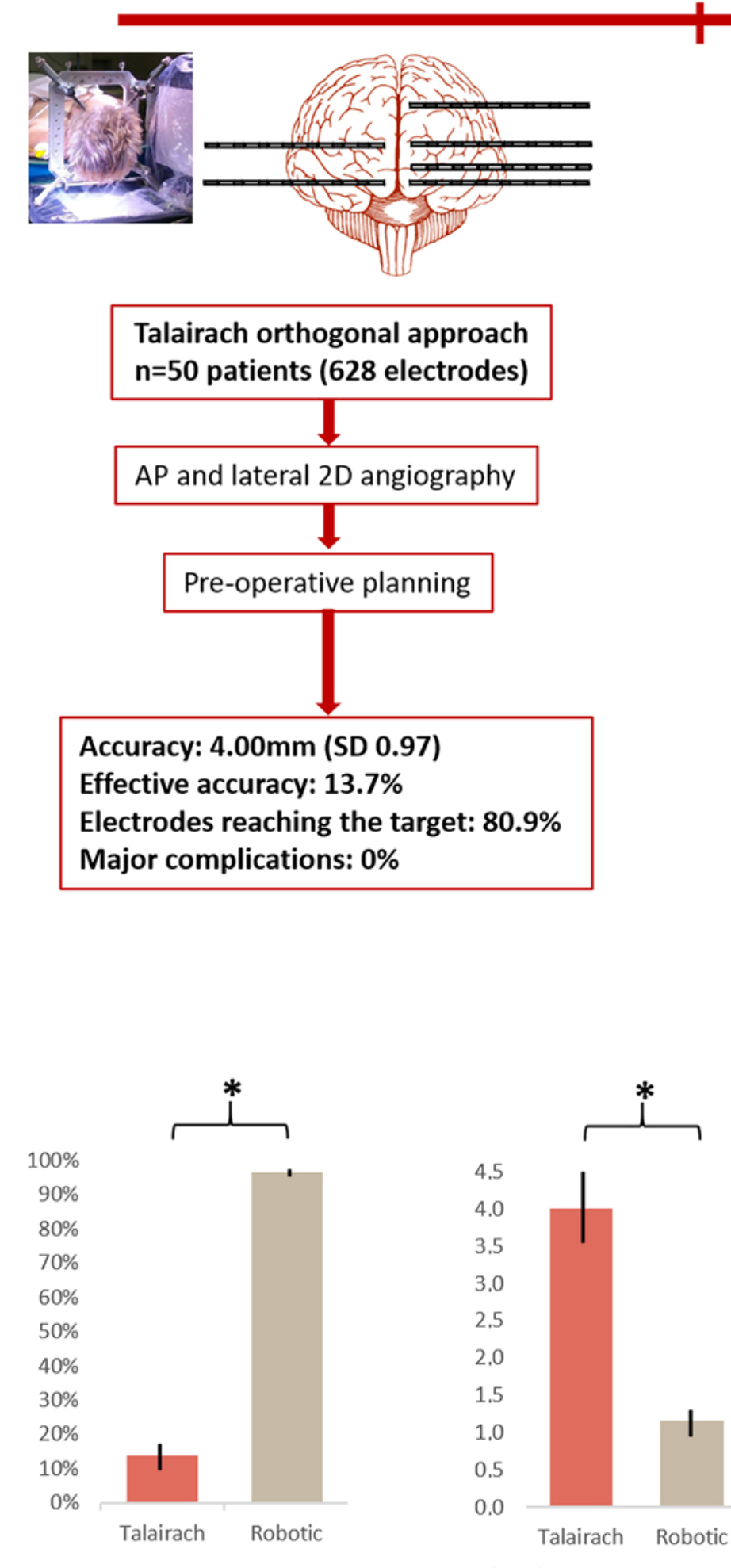

Effective accuracy (\%)

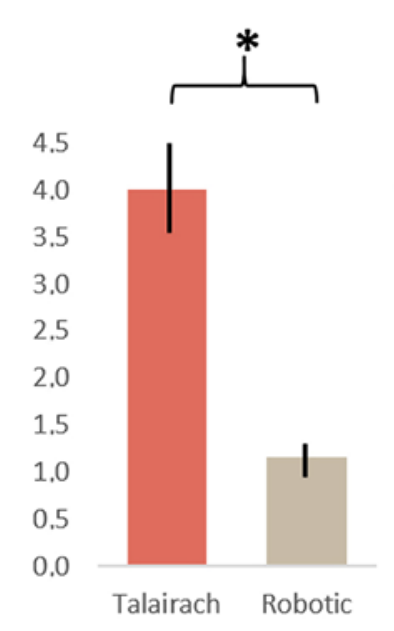

\section{Accuracy: $1.15 \mathrm{~mm}$ (SD 0.36)}

Effective accuracy: $96.5 \%$

Electrodes reaching the target: $\mathbf{9 8 . 6 \%}$

Major complications: $\mathbf{0 \%}$

FIG. 1. Flowchart showing the Talairach orthogonal approach (left) and the robotic 3D approach (right) with bar graphs showing effective and absolute accuracy of the robotic 3D approach versus the Talairach orthogonal procedure. Asterisks indicate a significant difference. Figure is available in color online only. 

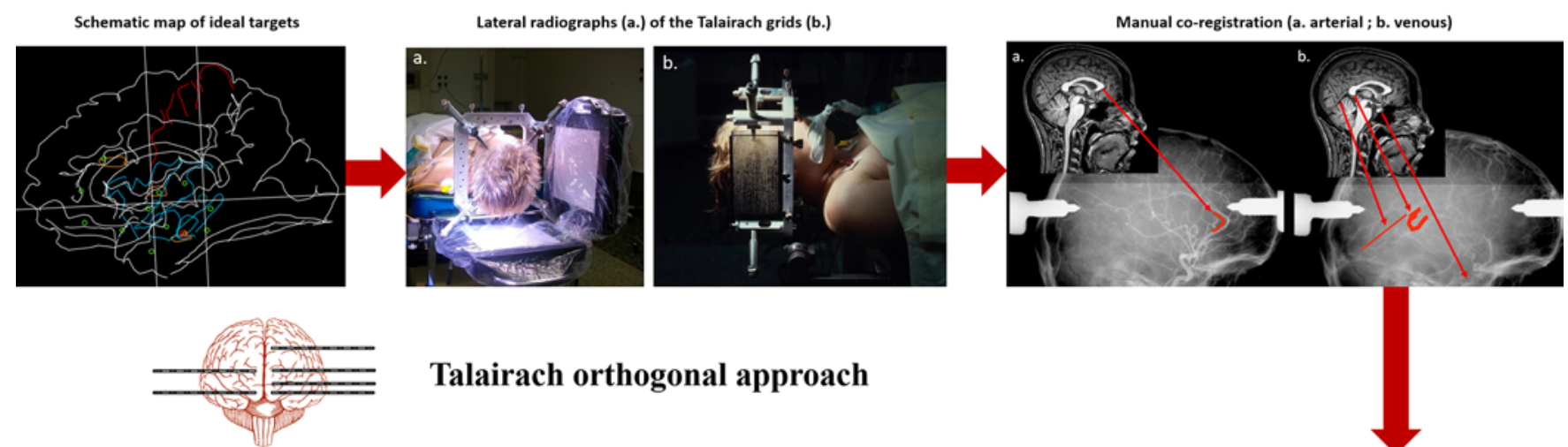

\section{Talairach orthogonal approach}

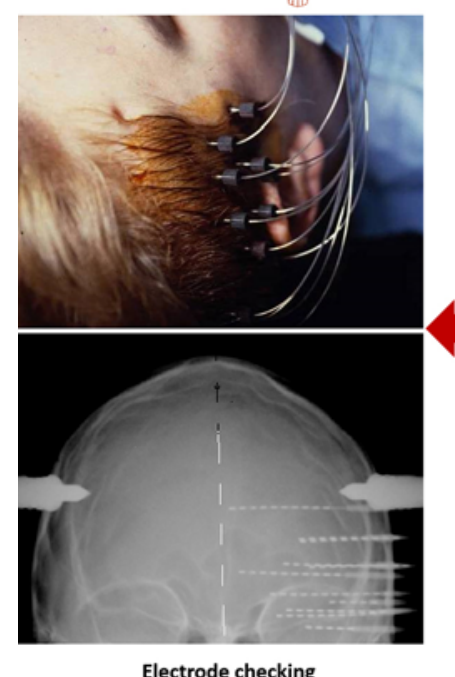

Electrode checking

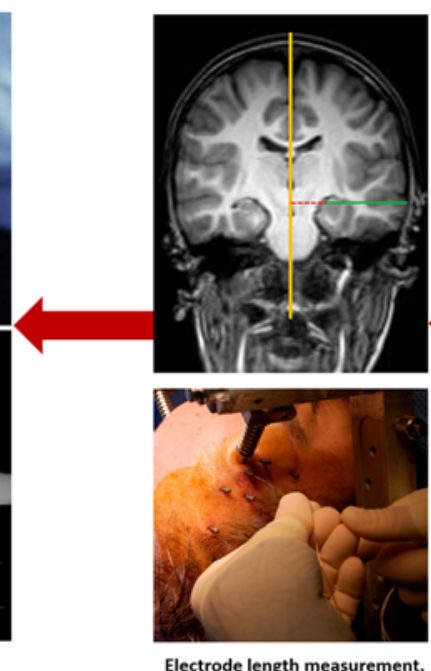

Electrode insertion

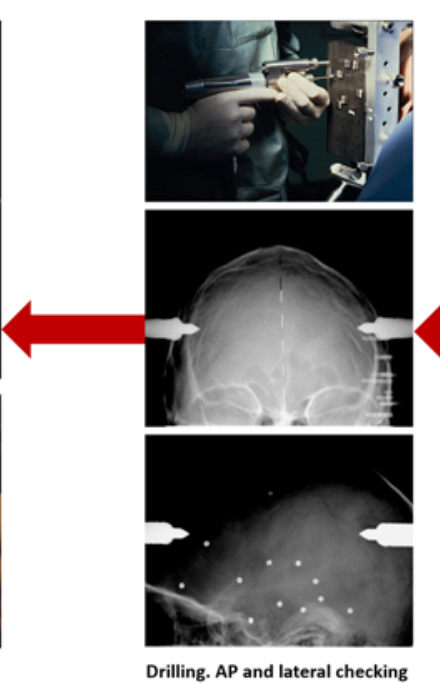

Drilling. AP and lateral checking of the Screw-in anchors

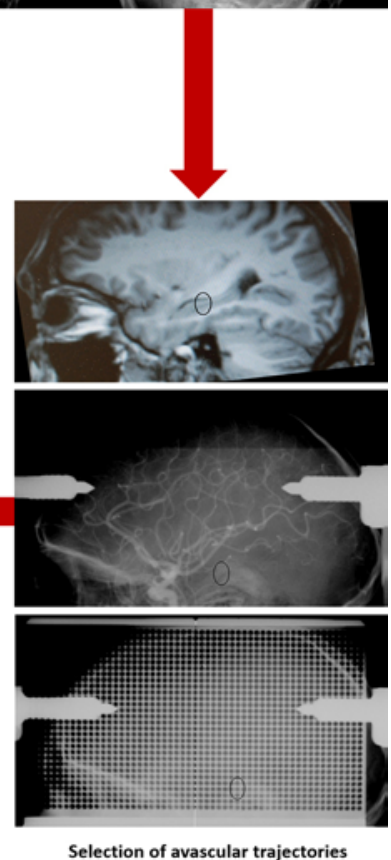

Selection of avascular trajectories

FIG. 2. Talairach orthogonal approach. Figure is available in color online only.

freedom, and with normalized mutual information as the cost function (Neuroinspire software version 5.0.721.1). All preplanned trajectories were then checked and modified as necessary (i.e., in case of vascular conflict or bone thickness $<2 \mathrm{~mm}$ ). The Neuromate robot (Renishaw PLC) was interlocked with the stereotactic frame and an external verification trajectory was made to check the mechanical accuracy of the robot before starting the procedure. The Neuromate has 5 axes of freedom (axes 1-3, $\pm 160^{\circ}$; axis $4, \pm 140^{\circ}$; axis $5, \pm 90^{\circ}$ ) and a frame-based precision, tested every 3 months, below $1.5 \mathrm{~mm}$.

For each planned electrode, the robot arm was then aligned to the planned trajectory. Using the arm's working channel, the scalp and the calvaria were then drilled and the dura opened by monopolar coagulation. The screwin anchors were screwed in place and their positions controlled using a second $\mathrm{O}$-arm for 3D imaging, with which the desirable electrode lengths were measured. If this control was satisfactory, electrodes (Microdeep) were inserted after a first path had been performed using a rigid smoothtipped stylus. Positioning of the electrodes was controlled using a third round of 3D imaging. Postoperative MRI was performed within 24 hours following the surgery.

\section{Evaluation Criteria}

The primary endpoint was the effective accuracy of the electrode locations. To be robust, an SEEG technique must on the one hand be accurate (ensuring vascular safety) and on the other hand reach the right anatomical target (ensuring diagnostic or therapeutic efficacy). The concept of effective accuracy, combining these 2 aspects, was hence developed for our primary outcome. To be effectively accurate, an electrode must never deviate from the planned trajectory by more than $2 \mathrm{~mm}$ and must reach the targeted anatomical structure (Fig. 4). Two millimeters corresponds, in the opinion of the authors, to the radius of a safety zone along trajectories in highly vascularized regions such as the insula, ${ }^{7}$ which are frequently involved in epileptic networks.

Our first secondary endpoint was the absolute precision defined by the Euclidian distance between the actual location of the electrode and the planned trajectory. ${ }^{10}$ This criterion is secondary because it is less clinically relevant than effective accuracy. Nevertheless, as this study is the first to compare the original Talairach procedure to a robotic 3D approach, we thought it appropriate to have a quantitative measurement of the accuracy as well. The Euclidian distance $\delta$ between the 2 points $\alpha$ and $\beta$ is

$$
\delta(\alpha, \beta)=\sqrt{\left(\alpha_{x}-\beta_{x}\right)^{2}+\left(\alpha_{y}-\beta_{y}\right)^{2}+\left(\alpha_{z}-\beta_{z}\right)^{2}} .
$$

The second secondary endpoint concerned the robotic 3D approach group only. In this procedure, presurgical 

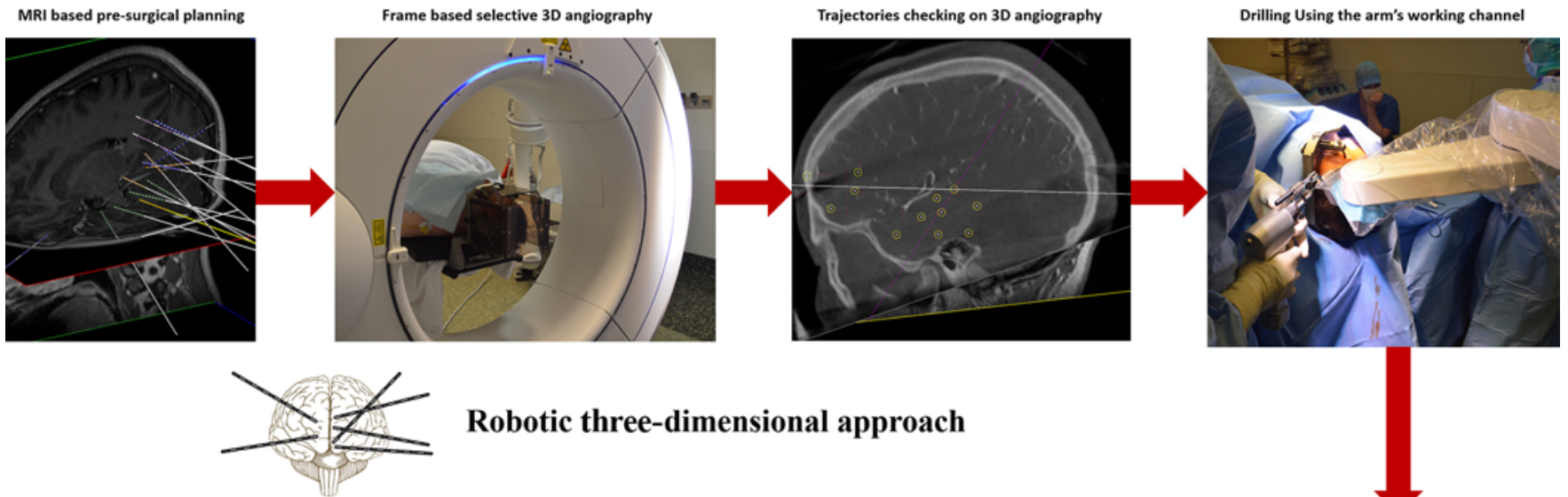

\section{Robotic three-dimensional approach}

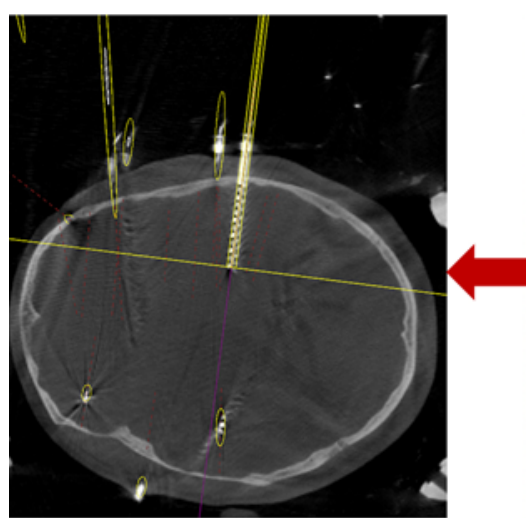

Electrode checking

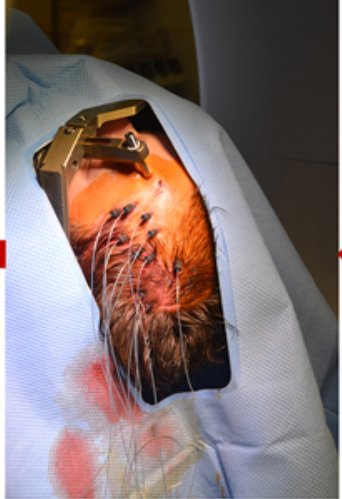

Electrode insertion

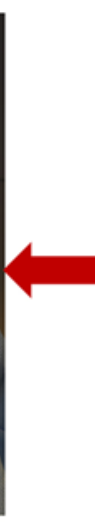

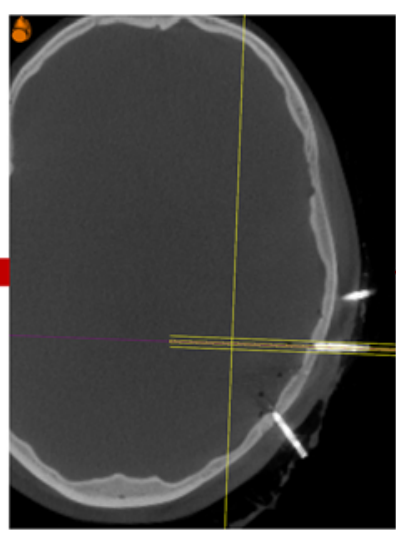

Accuracy checking based on screw-in anchors

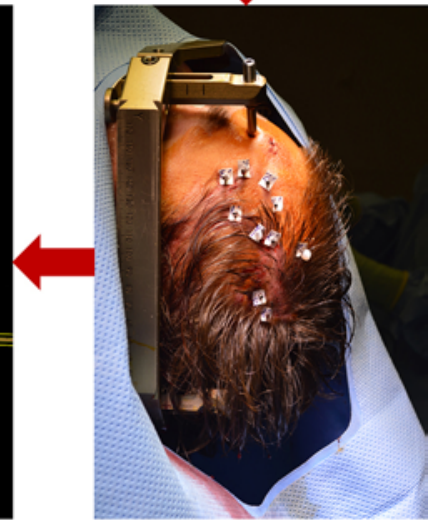

Screw-in anchors

FIG. 3. Robotic 3D approach. Figure is available in color online only.

planning based on the vascular anatomy explored by $\mathrm{T} 1$ gadolinium-enhanced MRI was performed prior to surgery to define the final trajectory based on the 3D framebased intraoperative angiography (Fig. 1). This comparison provided an opportunity to evaluate the actual reliability of the MRI-based presurgical planning. Therefore, the rate of T1 gadolinium-enhanced MRI-based trajectories having required a modification was studied.

Our third and last secondary endpoint was the safety of the procedures. This endpoint was defined by the occurrence of major (for which consequences may lead to death or neurological impairment) or minor side effects related to either procedure.

\section{Statistical Analysis}

Categorical variables were expressed as numbers (n) and percentages. When the distribution was normal, quantitative variables were expressed as mean \pm standard deviation, and when the distribution was not normal, as median with minimum and maximum. The Kolmogorov-Smirnov test was used to check the hypothesis of normal distribution of quantitative variables. Categorical variables were compared using the chi-square test, when applicable, or Fisher's exact test. After verification of equality of variances when data were normally distributed, quantitative variables were compared between groups using the Student t-test. In cases of nonverification of normality of dis- tribution, the nonparametric Wilcoxon test was used. The statistical tests were bilateral and the level of significance was set to 5\% ( $\mathrm{p}<0.05)$. Statistical analyses were conducted using Matlab R2017a and the Statistics and Machine Learning Toolbox (MathWorks, Inc.).

\section{Results}

From 2015 to 2017, 50 patients underwent SEEG procedures using a robotic 3D approach. The same number of patients who underwent procedures performed before 2015, using the Talairach approach, were selected for use as controls. The comparison of the 2 populations (Table 1) revealed a significantly higher number of electrodes implanted per patient in the Talairach approach group (mean $12.56 \pm 2.23$ electrodes) than in the robotic 3D approach group (mean $11.30 \pm 3.25$ electrodes) $(\mathrm{p}=0.008 ; 95 \% \mathrm{CI}$ $0.340-2.180 ; t=2.717$ ). Procedure duration (from the installation of the frame until its ablation) was compared between the Talairach approach (mean length $245.80 \pm$ 46.09 minutes) and the robotic 3D approach (mean length $239.80 \pm 66.70$ minutes) and found not to be significantly different $(p=0.298 ; 95 \% C I-5.3941$ to $17.3941 ; t=1.05)$.

A significant difference (Fig. 1) in terms of effective accuracy was found between the 2 groups ( $<<0.001 ; 95 \%$ CI -0.863 to $-0.781 ; \mathrm{t}=-39.92$ ). Among the 628 electrodes implanted according to the Talairach orthogonal proce- 


\section{Effective accuracy:}

1. Less than $2 \mathrm{~mm}$ deviation from the planned trajectory

2. Reaching the anatomical targeted structure

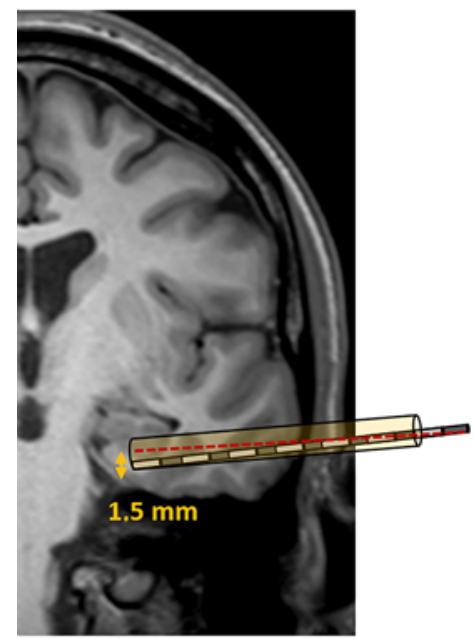

Effective accuracy not reached :

Despite a deviation inferior to $2 \mathrm{~mm}$, the target (hippocampus) is not reached.

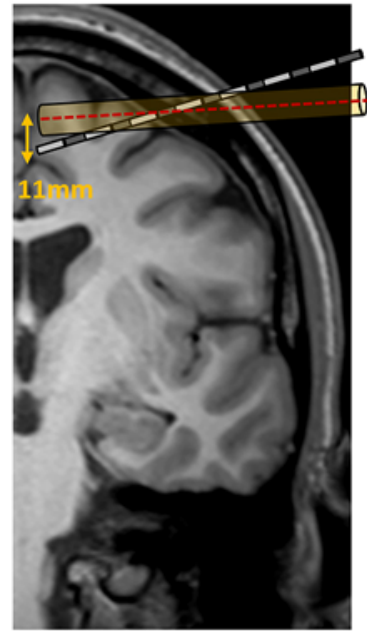

Effective accuracy not reached:

Despite the target being reached, the electrode has deviated by more than $2 \mathrm{~mm}$ from the planned trajectory

FIG. 4. Definition of effective accuracy. Figure is available in color online only.

dure, $86(13.7 \%)$ reached the criteria of effective accuracy. In the 3D robotic group, 545 of the 565 (96.5\%) electrodes reached these criteria. Interestingly, when analyzing the subcriterion of effective accuracy, we found that 508 of the $628(80.9 \%)$ electrodes implanted according to the Talairach orthogonal procedure and 557 of 565 (98.6\%) in the other group reached the anatomical target. This difference was significant $(\mathrm{p}<0.001)$.

The absolute accuracy was also significantly higher in the 3D robotic group (mean $1.15 \pm 0.36$ ) than in the orthogonal group (mean $4.00 \pm 0.97)(\mathrm{p}<0.001 ; 95 \% \mathrm{CI}$ $2.597-3.183 ; \mathrm{t}=19.73)$.

With regard to the reliability of presurgical planning using gadolinium-enhanced MRI, we observed that $43.8 \%$ of the trajectories had to be modified because of the presence of vessels, which were visualized within the $2-\mathrm{mm}$ radius safety zone around the trajectory on intraoperative frame-based 3D angiography (Fig. 1). The anatomical locations of the electrodes were a significant $(p<0.001)$ factor for angiography-based corrections; 85 of the 199 (42.7\%) frontal electrodes required a correction, as well as 28 of the $68(41.2 \%)$ parietal electrodes, 57 of the 262 (21.8\%) temporal electrodes, 70 of the $96(72.9 \%)$ insular electrodes, and 12 of the 32 (37.5\%) occipital electrodes. When the groups were compared, insular and frontal trajectories had a significantly higher rate of modification ( $p$ $<0.001$ and $p=0.04$, respectively), while temporal electrodes displayed a significantly lower $(p<0.001)$ need for trajectory modification than electrodes in other locations.

TABLE 1. Patient population characteristics

\begin{tabular}{lccc}
\hline & $\begin{array}{c}\text { Talairach } \\
\text { Procedure } \\
(n=50)\end{array}$ & $\begin{array}{c}\text { Robotic } \\
\text { Procedure } \\
(n=50)\end{array}$ & $\begin{array}{c}p \\
\text { Value }\end{array}$ \\
\hline Age in yrs, mean \pm SD & $29 \pm 11.4$ & $29.6 \pm 11.2$ & $0.881^{*}$ \\
\hline Sex, M/F & $27: 23$ & $34: 16$ & $0.219 \dagger$ \\
\hline No. of implanted electrodes & 628 & 565 & $0.008^{*}$ \\
\hline No. of bilateral implantations & 26 & 16 & $0.068 \dagger$ \\
\hline${ }^{*}$ Student t-test. & & & \\
$\dagger$ Chi-square test. & & &
\end{tabular}

Modification rates for occipital $(\mathrm{p}=0.183)$ and parietal ( $\mathrm{p}$ $=0.39$ ) locations were intermediate.

In both groups, no major complications occurred. Concerning minor complications, 1 patient in each group had electrode breakage in the days following the implantation (leading to loss of EEG signal from this electrode). After the Talairach orthogonal procedure, 1 patient was found to have a subdural fluid collection, for which we could not determine if it was a CSF hygroma or a chronic subdural hematoma (Fig. 5), and in the robotic 3D approach group, there was 1 case of asymptomatic acute pneumocephalus following the removal of electrodes. The difference in complication rates was not significant between the 2 groups.

\section{Discussion \\ Effective and Absolute Accuracy}

The concept of effective accuracy was introduced to take into account the specificities of SEEG in stereotaxy. In contrast to therapeutic stereotactic procedures, such as deep brain stimulation (DBS) procedures, in which the ability to reach a target, identical for all patients, correlates with the clinical outcome, SEEG is a diagnostic procedure that explores epileptic networks using multiple electrodes, different in all patients, and targets suspected epileptogenic foci as well as suspected propagation zones. SEEG results are then used in conjunction with other noninvasive investigations (EEG, EEG-functional MRI, PET, MEG) to delineate the seizure onset zone (the surgical target). Consequently, making a direct correlation between the absolute electrode accuracy and the clinical outcome is difficult. Therefore, this study focused on a more specific point, the ability of each electrode to target the desired anatomical structure through a safe (avascular) trajectory: the effective accuracy.

The robotic 3D approach displays a much higher accuracy, both effective ( $96.5 \%$ vs $13.7 \%$ ) and absolute (1.15 vs $4.00 \mathrm{~mm}$ ), than the orthogonal Talairach approach, highlighting the greater ability of the robotic 3D approach to reliably follow a planned trajectory (within a 2-mm radius) to a planned target.

Such a strong difference in results with the use of 2 different methods, found in a single center over a rather short period, seems to clearly demonstrate the intrinsic superiority of the robotic 3D approach and thus advocates that it be used rather than the traditional Talairach orthogonal methodology, especially for targets or trajectories within 


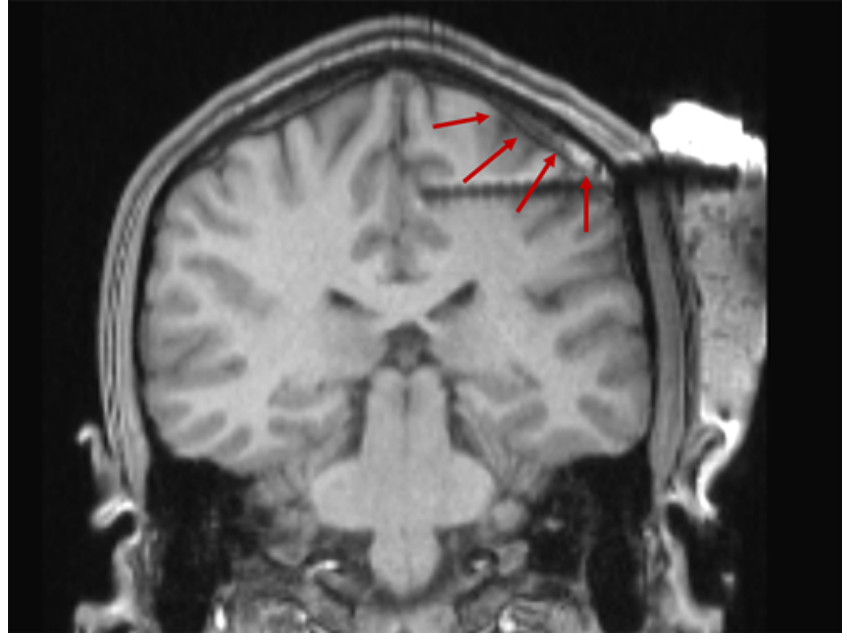

FIG. 5. Subdural fluid collection occurring in a patient after a Talairach orthogonal procedure (red arrows). Figure is available in color online only.

highly vascularized areas (such as the insula). Logical explanations can be found for these findings. Foremost, the robotic approach is based on a unique 3D stereotactic space, in which MRI and 3D angiography are fused together using a robust computerized algorithm, while in the Talairach orthogonal procedure, the stereotactic space is simply constituted by a 2D angiography on which selected 2D MR images are superimposed. Furthermore, the Talairach orthogonal procedure is performed using a manual coregistration of the stereotactic space (i.e., the framebased angiography) with the MRI. This coregistration largely relies on the anatomical skills of the surgeon and is a linear procedure with only 3 degrees of freedom (rotation, translation, and an enlargement factor), whereas the Neuroinspire software uses a rigid body registration with 6 degrees of freedom (3 rotations and 3 translations), and with normalized mutual symbolic information as the cost function. This latter type of nonlinear algorithm is considered more robust than the linear ones and is hence becoming increasingly available in CE- and FDA-approved surgical planning software. ${ }^{14}$ We believe that this difference in the stereotactic space constitutes the main factor explaining a significant difference in terms of effective and absolute accuracy between our 2 groups. A second contributing factor consists of a purely mechanical point. The Talairach orthogonal procedure uses strictly orthogonal trajectories, a feature that makes it more difficult to accurately reach some structures, in particular those close to the vertex or to the frontal or occipital pole, which require a more tangential approach to the skull. It is certain that drilling tangential to the bone can lead to a deviation of the screw-in anchor, which ultimately determines the trajectory of the electrode.

Absolute accuracy, although perhaps being less relevant for daily clinical practice than effective accuracy, was also significantly better in the robotic group. The accuracy of robotic procedures relies mainly on 2 aspects: $:^{13}$ the intrinsic accuracy of the robot itself and that of the coregistration/navigation software. The intrinsic accuracy of the Neuromate robot has already been evaluated for various procedures in neurosurgery, ${ }^{28}$ but only once before specifically for SEEG..$^{10}$ To the best of our knowledge, no SEEG study using a nonlinear coregistration algorithm has been previously published. The absolute accuracy of the new procedure described here $(1.15 \pm 0.36 \mathrm{~mm})$ is higher than that of other robotic procedures reported using the Neuromate $(2.04 \pm 1.31 \mathrm{~mm})^{10}$ or the ROSA robot $(3.3 \mathrm{~mm} ; 2.09$ $\pm 1.06-3.72 \mathrm{~mm}),{ }^{11,25}$ as well as frameless neuronavigation procedures $(3.6 \pm 3.85 \mathrm{~mm} ; 2.96 \pm 1.49 \mathrm{~mm}){ }^{8,24}$

However, it must be said that the less favorable results, in terms of effective and absolute accuracy, of the original Talairach technique must not lead to the conclusion that the technique is unsafe. The Talairach orthogonal procedure had initially been developed in $1962^{27}$ to perform implantations based on the eponym atlas, ventriculography, and angiography (MRI was not available). Despite this limitation, the technique has proven its ability to obtain long-lasting intracranial EEG recordings with a very high level of safety $\left(0.3 \%\right.$ major complication rate). ${ }^{22}$ The main relevance of our findings is that a combined MRI-3D angiography, robot-assisted approach is more accurate than a pure orthogonal methodology relying on AP and lateral angiography images coupled with manually coregistered selected MR images for targeting. When only the ability to reach a relevant anatomical target, whatever geometrical accuracy, is taken into account, the difference between the 2 techniques is still significantly unfavorable to the Talairach orthogonal procedure, but the difference (17.7\%) is not so strong.

Another interesting finding, not related to the accuracy itself, is that the Talairach approach group had more implanted electrodes per patient (mean $12.56 \pm 2.23$ ) than the robotic 3D approach group (mean $11.30 \pm 3.25$ ). This difference could be due to the fact that, in the robotic 3D approach, the possible trajectories of electrodes are infinite, whereas the orthogonal approach only allows a finite number of trajectories within a metallic grid, all of which are in an orthogonal axis to this grid. Therefore, the 3D robotic approach may allow the same level of sampling with fewer electrodes, by permitting the recording of 2 or more targets with the same electrode, which constitutes one more argument in favor of the use of this technique. However, another explanation could be that the growing experience of the epileptologists and the increasing performances of phase I investigations led to a decrease in the number of electrodes needed to investigate epileptic networks.

\section{Reliability of MRI-Based Trajectory Planning}

Major advances have occurred in vascular imaging since the first descriptions of SEEG. Angio-CT or T1 gadolinium-enhanced MRI have been proposed as noninvasive alternatives to traditional angiography in planning avascular trajectories. However, no controlled study has been done to compare modern imaging techniques to the gold standard (the angiography) in SEEG. Data from different case series suggest differences in safety between the 2 approaches. For instance, the $0.3 \%$ bleeding rate observed by Munari (angiography-based procedure) ${ }^{22}$ contrasts with the recent $3.7 \%{ }^{20}$ or $12.7 \%{ }^{25}$ bleeding rates observed in groups using angio-MR. Nevertheless, making conclusions based on 
published case series is hazardous, as many other factors may contribute to this variability (type of electrode experience of the team, robotic assistance, use of a frame-based procedure, methods for coregistration). Short of a randomized controlled trial, the design of this study nonetheless provided the authors with the opportunity to evaluate, in each patient, the ability to plan avascular trajectories using only the presurgical T1 gadolinium-enhanced MRI scan. The frame-based superimposed 3D angiography was then used as the gold standard for vascular safety. Therefore, we provide this first comparison between common carotid selective 3D angiography and T1 gadolinium-enhanced MRI scans for the planning of safe avascular SEEG trajectories. The frame-based intraoperative 3D angiography has an intrinsic accuracy advantage over the presurgical MRI, being contemporary to the time of implantation and free of geometrical distortions due to potential magnetic field inhomogeneity. The high rate $(43.8 \%)$ of trajectory modification because of the presence of vessels (on angiography) within the $2-\mathrm{mm}$ radius of the MRI-planned trajectory (especially those reaching the insula through its dense vascular environment [72.9\%]) is a strong argument to continue performing angiographies for SEEG. The authors observed that, in some cases, the problematic vessel had been seen on the MRI, but followed a slightly different path. One can assume that the actual vessel localization is more reliably represented by the same-day, frame-based $3 \mathrm{D}$ angiography than by the MRI performed a few days before (due to shift, distortion, and coregistration issues discussed earlier). In other cases, a small vessel, not seen on MRI, was visible on the 3D angiography. Therefore, this finding highlights the ability of the robotic 3D approach to safely plan trajectories using a 2-mm avascular corridor, whereas an MRI-based procedure would require a larger safety zone to palliate for vessel deviation (due to shift, distortion, and coregistration issues), which may lead to difficulties in finding trajectories in dense vascular areas, frequently involved in epileptic networks, and would also not address the risk of smaller-caliber vessels (unseen on MRI) being injured. We realize that the actual safety of the initially planned (MRI-only) trajectories cannot be quantified since these trajectories were often modified after the 3D angiography was added, but it is assumed that passing an electrode within $2 \mathrm{~mm}$ of an angiographically visible vessel carries a bleeding risk.

Current CT angiography techniques using intravenous contrast injection have demonstrated small-vessel visualization abilities comparable to those of digital subtraction angiography, without the potential risks associated with carotid artery catheterization. The protocol described in this study used O-arm 3D acquisition but still relied on a common carotid injection. No complications of arterial puncture and catheterization were recorded in this study; however, there is a documented risk to the procedure. The use of a same-day, frame-based, 3D angiography with intravenous contrast injection, in order to retain the accuracy advantages of the contemporary frame-based imaging, while mitigating vascular risks, would likely yield comparable results to the robotic $3 \mathrm{D}$ group.

It is difficult to make any conclusion regarding the comparative safety of the 2 procedures, as the size of our stud- ied population was limited in relation to the very low rate of major complications. The prevalence of severe complications related to SEEG procedures has been reported to range between $0.30 \%$ and $1.70 \%$ in the literature. ${ }^{7,21,22}$ Therefore, the absence of any major complications related to the 100 procedures reported here is not surprising and allows us to conclude that both techniques seem to have a low rate of major complications, in accordance with previous reports. The similar and very low rate of complications in SEEG procedures despite the variability in methods and documented accuracy $7,8,10,11,18,20$ is likely explained by the experience of the different groups with their implantation method of choice and its limits: electrodes are only implanted if the trajectory is deemed safe, even if it means omitting a planned target. SEEG implantations performed using techniques with lower documented accuracy (frameless, MRI-based) likely entail planned trajectories with wider avascular corridors and likely avoid dense vascular areas such as the insula, or use less vascular parasagittal approaches to obtain recordings from this region.

\section{Conclusions}

The robotic 3D approach has a higher effective accuracy and a higher absolute accuracy than the Talairach orthogonal procedure for SEEG. An intraoperative, frame-based, 3D angiography method provides a safety advantage over T1 gadolinium-enhanced MRI alone for trajectory planning by providing more detailed vascular information, acquired in the exact intraoperative position of the patient (not subject to coregistration or field distortion errors). To our knowledge, this frame-based, 3D angiographic, robotassisted procedure provides the best reported accuracy for SEEG electrode implantation.

\section{References}

1. Apra C, Bourdillon P, Lévêque M: Surgical techniques: when brain bullets met crowdfunding. Nature 530:160, 2016

2. Bourdillon P, Apra C, Lévêque M: First clinical use of stereotaxy in humans: the key role of $\mathrm{x}$-ray localization discovered by Gaston Contremoulins. J Neurosurg 128:932-937, 2018

3. Bourdillon P, Apra C, Lévêque M, Vinckier F: Neuroplasticity and the brain connectome: what can Jean Talairach's reflections bring to modern psychosurgery? Neurosurg Focus 43(3):E11, 2017

4. Bourdillon P, Isnard J, Catenoix H, Montavont A, Rheims S, Ryvlin P, et al: Stereo-electro-encephalography-guided radiofrequency thermocoagulation: from in vitro and in vivo data to technical guidelines. World Neurosurg 94:73-79, 2016

5. Bourdillon P, Isnard J, Catenoix H, Montavont A, Rheims $\mathrm{S}$, Ryvlin P, et al: Stereo electroencephalography-guided radiofrequency thermocoagulation (SEEG-guided RF-TC) in drug-resistant focal epilepsy: results from a 10-year experience. Epilepsia 58:85-93, 2017

6. Bourdillon P, Lévêque M, Apra C: Second World War: Paris neurosurgeon's map outwitted Nazis. Nature 547:281, 2017

7. Bourdillon P, Ryvlin P, Isnard J, Montavont A, Catenoix H, Mauguière F, et al: Stereotactic electroencephalography is a safe procedure, including for insular implantations. World Neurosurg 99:353-361, 2017

8. Budke M, Avecillas-Chasin JM, Villarejo F: Implantation of depth electrodes in children using VarioGuide frameless navigation system: technical note. Oper Neurosurg (Hagerstown, Md) 15:302-309, 2018 
9. Cardinale F: Talairach methodology in the era of 3D multimodal imaging: "the song remains the same," but catchier, and therefore more helpful for clinical decision making and surgical planning in epilepsy surgery. Epilepsia 56:976-977, 2015

10. Cardinale F, Cossu M, Castana L, Casaceli G, Schiariti MP, Miserocchi A, et al: Stereoelectroencephalography: surgical methodology, safety, and stereotactic application accuracy in 500 procedures. Neurosurgery 72:353-366, 2013

11. Châtillon CE, Mok K, Hall J, Olivier A: Comparative study of manual versus robot-assisted frameless stereotaxy for intracranial electrode implantation. Presented at the 65th AES Annual Meeting, Baltimore, 2011 (Poster) (http://www. medtechsurgical.com/Pressroom/Peer-review) [Accessed August 23, 2018]

12. Cossu M, Fuschillo D, Cardinale F, Castana L, Francione S, Nobili L, et al: Stereo-EEG-guided radio-frequency thermocoagulations of epileptogenic grey-matter nodular heterotopy. J Neurol Neurosurg Psychiatry 85:611-617, 2014

13. Fomenko A, Serletis D: Robotic stereotaxy in cranial neurosurgery: a qualitative systematic review. Neurosurgery 83:642-650, 2018

14. Geevarghese R, O'Gorman Tuura R, Lumsden DE, Samuel M, Ashkan K: Registration accuracy of CT/MRI fusion for localisation of deep brain stimulation electrode position: an imaging study and systematic review. Stereotact Funct Neurosurg 94:159-163, 2016

15. Giller CA, Mornet P, Moreau JF: The first formulation of image-based stereotactic principles: the forgotten work of Gaston Contremoulins. J Neurosurg 127:1426-1435, 2017

16. Guénot M: SEEG electrodes implantation using flat-panel $\mathrm{x}$-ray detectors and robot, in Scarabin JM (ed): Stereotaxy and Epilepsy Surgery. Montrouge, France: John Libbey Eurotext, 2012, pp 219-244

17. Guénot M, Isnard J, Ryvlin P, Fischer C, Mauguière F, Sindou M: SEEG-guided RF thermocoagulation of epileptic foci: feasibility, safety, and preliminary results. Epilepsia 45:1368-1374, 2004

18. Guenot M, Isnard J, Ryvlin P, Fischer C, Ostrowsky K, Mauguiere F, et al: Neurophysiological monitoring for epilepsy surgery: the Talairach SEEG method. StereoElectroEncephaloGraphy. Indications, results, complications and therapeutic applications in a series of 100 consecutive cases. Stereotact Funct Neurosurg 77:29-32, 2001

19. Leksell L: The stereotaxic method and radiosurgery of the brain. Acta Chir Scand 102:316-319, 1951

20. Mathon B, Clemenceau S, Hasboun D, Habert MO, Belaid $\mathrm{H}$, Nguyen-Michel VH, et al: Safety profile of intracranial electrode implantation for video-EEG recordings in drugresistant focal epilepsy. J Neurol 262:2699-2712, 2015
21. Mullin JP, Shriver M, Alomar S, Najm I, Bulacio J, Chauvel P, et al: Is SEEG safe? A systematic review and meta-analysis of stereo-electroencephalography-related complications. Epilepsia 57:386-401, 2016

22. Munari C: Depth electrode implantation at Hôpital Sainte Anne, Paris, in Engel J Jr (ed): Surgical Treatment of the Epilepsies. New York: Raven Press, 1987, pp 583-588

23. Narizzano M, Arnulfo G, Ricci S, Toselli B, Tisdall M, Canessa A, et al: SEEG assistant: a 3DSlicer extension to support epilepsy surgery. BMC Bioinformatics 18:124, 2017

24. Nowell M, Rodionov R, Diehl B, Wehner T, Zombori G, Kinghorn J, et al: A novel method for implementation of frameless StereoEEG in epilepsy surgery. Neurosurgery 10 (Suppl 4):525-534, 2014

25. Ollivier I, Behr C, Cebula H, Timofeev A, Benmekhbi M, Valenti MP, et al: Efficacy and safety in frameless robotassisted stereo-electroencephalography (SEEG) for drugresistant epilepsy. Neurochirurgie 63:286-290, 2017

26. Spiegel EA, Wycis HT, Marks M, Lee AJ: Stereotaxic apparatus for operations on the human brain. Science 106:349350,1947

27. Talairach J, Bancaud J, Bonis A, Szikla G, Tournoux P: Functional stereotaxic exploration of epilepsy. Confin Neurol 22:328-331, 1962

28. Varma TRK, Eldridge P: Use of the NeuroMate stereotactic robot in a frameless mode for functional neurosurgery. Int J Med Robot 2:107-113, 2006

\section{Disclosures}

The authors report no conflict of interest concerning the materials or methods used in this study or the findings specified in this paper.

\section{Author Contributions}

Conception and design: Bourdillon, Châtillon, Guénot. Acquisition of data: Bourdillon, Châtillon, Moles, Guénot. Analysis and interpretation of data: Bourdillon, Châtillon, Guénot. Drafting the article: Bourdillon. Critically revising the article: Châtillon, Moles, Rheims, Catenoix, Montavont, Ostrowsky-Coste, Boulogne, Isnard, Guénot. Approved the final version of the manuscript on behalf of all authors: Bourdillon. Statistical analysis: Bourdillon. Study supervision: Guénot.

\section{Correspondence}

Pierre Bourdillon: P. Wertheimer Hospital, Hospices Civils de Lyon, Bron, France. pierre.bourdillon@neurochirurgie.fr. 